\title{
Recurrence formulae for Apostol-Bernoulli and Apostol-Euler polynomials
}

Yuan $\mathrm{He}^{*}$ and Chunping Wang

\section{"Correspondence:}

hyyhe@yahoo.com.cn

Faculty of Science, Kunming

University of Science and

Technology, Kunming, Yunnan

650500, People's Republic of China

\begin{abstract}
In this paper, using generating functions and combinatorial techniques, we extend Agoh and Dilcher's quadratic recurrence formula for Bernoulli numbers in (Agoh and Dilcher in J. Number Theory 124:105-122, 2007) to Apostol-Bernoulli and Apostol-Euler polynomials and numbers.

MSC: 11B68;05A19
\end{abstract}

Keywords: Apostol-Bernoulli polynomials and numbers; Apostol-Euler polynomials and numbers; recurrence formula

\section{Introduction}

The classical Bernoulli polynomials $B_{n}(x)$ and Euler polynomials $E_{n}(x)$ have played important roles in many branches of mathematics such as number theory, combinatorics, special functions and analysis, and they are usually defined by means of the following generating functions:

$$
\frac{t e^{x t}}{e^{t}-1}=\sum_{n=0}^{\infty} B_{n}(x) \frac{t^{n}}{n !} \quad(|t|<2 \pi), \quad \frac{2 e^{x t}}{e^{t}+1}=\sum_{n=0}^{\infty} E_{n}(x) \frac{t^{n}}{n !} \quad(|t|<\pi) .
$$

In particular, $B_{n}=B_{n}(0)$ and $E_{n}=2^{n} E_{n}(1 / 2)$ are called the classical Bernoulli numbers and Euler numbers, respectively. Numerous interesting properties for these polynomials and numbers have been explored; see, for example, [1-3].

Recently, using some relationships involving Bernoulli numbers, Agoh and Dilcher [4] extended Euler's well-known quadratic recurrence formula on the classical Bernoulli numbers

$$
\left(B_{0}+B_{0}\right)^{n}=\sum_{i=0}^{n}\left(\begin{array}{c}
n \\
i
\end{array}\right) B_{i} B_{n-i}=-n B_{n-1}-(n-1) B_{n} \quad(n \geq 1)
$$

to obtain an explicit expression for $\left(B_{k}+B_{m}\right)^{n}$ with arbitrary non-negative integers $k, m$, $n$ and $k$ and $m$ not both zero as follows:

$$
\begin{aligned}
\left(B_{k}+B_{m}\right)^{n} & =\sum_{i=0}^{n}\left(\begin{array}{l}
n \\
i
\end{array}\right) B_{k+i} B_{m+n-i} \\
& =-\frac{k ! m !(n+\delta(k, m)(m+k+1))}{(m+k+1) !} B_{m+n+k}
\end{aligned}
$$

(c) 2012 He and Wang; licensee Springer. This is an Open Access article distributed under the terms of the Creative Commons Attribution License (http://creativecommons.org/licenses/by/2.0), which permits unrestricted use, distribution, and reproduction in any medium, provided the original work is properly cited. 


$$
\begin{aligned}
& +\sum_{i=1}^{m+k}(-1)^{i} \frac{B_{m+k+1-i}}{m+k+1-i}\left\{(-1)^{k}\left(\begin{array}{c}
k+1 \\
i
\end{array}\right)\left(\frac{k+1-i}{k+1} n-\frac{i m}{k+1}\right)\right. \\
& \left.+(-1)^{m}\left(\begin{array}{c}
m+1 \\
i
\end{array}\right)\left(\frac{m+1-i}{m+1} n-\frac{i k}{m+1}\right)\right\} B_{n+i-1},
\end{aligned}
$$

where $\delta(k, m)=0$ when $k=0$ or $m=0$, and $\delta(k, m)=1$ otherwise. As further applications, Agoh and Dilcher [4] derived some new types of recurrence formulae on the classical Bernoulli numbers and showed that the values of $B_{6 n}$ depend only on $B_{2 n}, B_{2 n+2}, \ldots, B_{4 n}$ for a positive integer $n$ and, similarly, for $B_{6 n+2}$ and $B_{6 n+4}$.

In this paper, using generating functions and combinatorial techniques, we extend the above mentioned Agoh and Dilcher quadratic recurrence formula for Bernoulli numbers to Apostol-Bernoulli and Apostol-Euler polynomials and numbers. These results also lead to some known ones related to the formulae on products of the classical Bernoulli and Euler polynomials and numbers stated in Nielsen's classical book [5].

\section{Preliminaries and known results}

We first recall the Apostol-Bernoulli polynomials which were introduced by Apostol [6] (see also Srivastava [7] for a systematic study) in order to evaluate the value of the HurwitzLerch zeta function. For simplicity, we here start with the Apostol-Bernoulli polynomials $\mathcal{B}_{n}^{(\alpha)}(x ; \lambda)$ of (real or complex) order $\alpha$ given by Luo and Srivastava [8]

$$
\left(\frac{t}{\lambda e^{t}-1}\right)^{\alpha} e^{x t}=\sum_{n=0}^{\infty} \mathcal{B}_{n}^{(\alpha)}(x ; \lambda) \frac{t^{n}}{n !} \quad\left(|t+\log \lambda|<2 \pi ; 1^{\alpha}:=1\right) .
$$

Especially the case $\alpha=1$ in (2.1) gives the Apostol-Bernoulli polynomials which are denoted by $\mathcal{B}_{n}(x ; \lambda)=\mathcal{B}_{n}^{(1)}(x ; \lambda)$. Moreover, $\mathcal{B}_{n}(\lambda)=\mathcal{B}_{n}(0 ; \lambda)$ are called the Apostol-Bernoulli numbers. Further, Luo [9] introduced the Apostol-Euler polynomials $\mathcal{E}_{n}^{(\alpha)}(x ; \lambda)$ of order $\alpha$ :

$$
\left(\frac{2}{\lambda e^{t}+1}\right)^{\alpha} e^{x t}=\sum_{n=0}^{\infty} \mathcal{E}_{n}^{(\alpha)}(x ; \lambda) \frac{t^{n}}{n !} \quad\left(|t+\log \lambda|<\pi ; 1^{\alpha}:=1\right) .
$$

The Apostol-Euler polynomials $\mathcal{E}_{n}(x ; \lambda)$ and Apostol-Euler numbers $\mathcal{E}_{n}(\lambda)$ are given by $\mathcal{E}_{n}(x ; \lambda)=\mathcal{E}_{n}^{(1)}(x ; \lambda)$ and $\mathcal{E}_{n}(\lambda)=2^{n} \mathcal{E}_{n}(1 / 2 ; \lambda)$, respectively. Obviously, $\mathcal{B}_{n}(x ; \lambda)$ and $\mathcal{E}_{n}(x ; \lambda)$ reduce to $B_{n}(x)$ and $E_{n}(x)$ when $\lambda=1$. Several interesting properties for Apostol-Bernoulli and Apostol-Euler polynomials and numbers have been presented in [10-17]. Next we give some basic properties for Apostol-Bernoulli and Apostol-Euler polynomials of order $\alpha$ as stated in $[8,9]$.

Proposition 2.1 Differential relations of the Apostol-Bernoulli and Apostol-Euler polynomials of order $\alpha$ :for non-negative integers $k$ and $n$ with $0 \leq k \leq n$,

$$
\frac{\partial^{k}}{\partial x^{k}}\left\{\mathcal{B}_{n}^{(\alpha)}(x ; \lambda)\right\}=\frac{n ! \mathcal{B}_{n-k}^{(\alpha)}(x ; \lambda)}{(n-k) !}, \quad \frac{\partial^{k}}{\partial x^{k}}\left\{\mathcal{E}_{n}^{(\alpha)}(x ; \lambda)\right\}=\frac{n ! \mathcal{E}_{n-k}^{(\alpha)}(x ; \lambda)}{(n-k) !} .
$$

Proposition 2.2 Difference equations of the Apostol-Bernoulli and Apostol-Euler polynomials of order $\alpha$ : for a positive integer $n$,

$$
\lambda \mathcal{B}_{n}^{(\alpha)}(x+1 ; \lambda)-\mathcal{B}_{n}^{(\alpha)}(x ; \lambda)=n \mathcal{B}_{n-1}^{(\alpha-1)}(x ; \lambda) \quad\left(\mathcal{B}_{n}^{(0)}(x ; \lambda)=x^{n}\right)
$$


and

$$
\lambda \mathcal{E}_{n}^{(\alpha)}(x+1 ; \lambda)+\mathcal{E}_{n}^{(\alpha)}(x ; \lambda)=2 \mathcal{E}_{n}^{(\alpha-1)}(x ; \lambda) \quad\left(\mathcal{E}_{n}^{(0)}(x ; \lambda)=x^{n}\right) .
$$

Proposition 2.3 Addition theorems of the Apostol-Bernoulli and Apostol-Euler polynomials of order $\alpha$ : for a suitable parameter $\beta$ and a non-negative integer $n$,

$$
\mathcal{B}_{n}^{(\alpha+\beta)}(x+y ; \lambda)=\sum_{i=0}^{n}\left(\begin{array}{c}
n \\
i
\end{array}\right) \mathcal{B}_{i}^{(\alpha)}(x ; \lambda) \mathcal{B}_{n-i}^{(\beta)}(y ; \lambda) \quad\left(\mathcal{B}_{n}^{(0)}(x ; \lambda)=x^{n}\right),
$$

and

$$
\mathcal{E}_{n}^{(\alpha+\beta)}(x+y ; \lambda)=\sum_{i=0}^{n}\left(\begin{array}{c}
n \\
i
\end{array}\right) \mathcal{E}_{i}^{(\alpha)}(x ; \lambda) \mathcal{E}_{n-i}^{(\beta)}(y ; \lambda) \quad\left(\mathcal{E}_{n}^{(0)}(x ; \lambda)=x^{n}\right) .
$$

Proposition 2.4 Complementary addition theorems of the Apostol-Bernoulli and ApostolEuler polynomials of order $\alpha$ : for a non-negative integer $n$,

$$
\mathcal{B}_{n}^{(\alpha)}(\alpha-x ; \lambda)=\frac{(-1)^{n}}{\lambda^{\alpha}} \mathcal{B}_{n}^{(\alpha)}\left(x ; \lambda^{-1}\right), \quad \mathcal{E}_{n}^{(\alpha)}(\alpha-x ; \lambda)=\frac{(-1)^{n}}{\lambda^{\alpha}} \mathcal{E}_{n}^{(\alpha)}\left(x ; \lambda^{-1}\right) .
$$

Setting $\alpha=1, \beta=0$ and $\alpha=\lambda=1, \beta=0$ in the formulae (2.3) to (2.8), we immediately get the corresponding formulae for the Apostol-Bernoulli and Apostol-Euler polynomials, and the classical Bernoulli and Euler polynomials, respectively. It is worth noting that the cases $\alpha=\lambda=1$ in Proposition 2.4 are also called the symmetric distributions of the classical Bernoulli and Euler polynomials. The above propositions will be very useful to investigate the quadratic recurrence formulae for the Apostol-Bernoulli and Apostol-Euler polynomials in the next two sections.

\section{Recurrence formulae for Apostol-Bernoulli polynomials}

In what follows, we shall always denote by $\delta_{1, \lambda}$ the Kronecker symbol which is defined by $\delta_{1, \lambda}=0$ or 1 according to $\lambda \neq 1$ or $\lambda=1$, and also denote $\mathcal{B}_{-n}(x ; \lambda)=\mathcal{E}_{-n}(x ; \lambda)=0$ for any positive integer $n$. Before stating the quadratic recurrence formula for the Apostol-Bernoulli polynomials, we begin with a summation formula concerning the quadratic recurrence of the Apostol-Bernoulli polynomials.

Theorem 3.1 Let $k, m, n$ be non-negative integers. Then

$$
\begin{aligned}
\sum_{i=0}^{n}\left(\begin{array}{c}
n \\
i
\end{array}\right)\left(\mathcal{B}_{i}(x ; \mu)+\mathcal{B}_{m+n-i}(y ; \lambda \mu)\right)^{k} \\
=-\delta_{1, \lambda} \frac{n+k}{m+1} \mathcal{B}_{m+n+k}(x+y ; \lambda \mu) \\
\quad+\mathcal{B}_{m}(y ; \lambda) \mathcal{B}_{n+k}(x+y ; \mu)+\frac{n+k}{m+1} \mathcal{B}_{m+1}(y ; \lambda) \mathcal{B}_{n+k-1}(x+y ; \mu) \\
\quad+(n+k) \sum_{i=0}^{m}\left(\begin{array}{c}
m \\
i
\end{array}\right)(-1)^{m+i} \mathcal{B}_{n+k+i-1}(x+y ; \lambda \mu) \frac{\mathcal{B}_{m+1-i}\left(x ; \lambda^{-1}\right)}{m+1-i} .
\end{aligned}
$$


Proof Multiplying both sides of the identity

$$
\frac{1}{\lambda e^{u}-1} \frac{1}{\mu e^{v}-1}=\left(\frac{\lambda e^{u}}{\lambda e^{u}-1}+\frac{1}{\mu e^{v}-1}\right) \frac{1}{\lambda \mu e^{u+v}-1}
$$

by $v(u+v) e^{x v+y(u+v)}$ yields

$$
\frac{v e^{x v}}{\mu e^{v}-1} \frac{(u+v) e^{y(u+v)}}{\lambda \mu e^{u+v}-1}=(u+v) \frac{e^{y u}}{\lambda e^{u}-1} \frac{v e^{(x+y) v}}{\mu e^{v}-1}-\lambda v \frac{e^{(1-x) u}}{\lambda e^{u}-1} \frac{(u+v) e^{(x+y)(u+v)}}{\lambda \mu e^{u+v}-1} .
$$

Making $k$-times derivative for the above identity (3.3) with respect to $v$, with the help of the Leibniz rule, we obtain

$$
\begin{aligned}
& \sum_{j=0}^{k}\left(\begin{array}{c}
k \\
j
\end{array}\right) \frac{\partial^{j}}{\partial v^{j}}\left(\frac{v e^{x v}}{\mu e^{v}-1}\right) \frac{\partial^{k-j}}{\partial v^{k-j}}\left(\frac{(u+v) e^{y(u+v)}}{\lambda \mu e^{u+v}-1}\right) \\
&=\frac{u e^{y u}}{\lambda e^{u}-1} \frac{\partial^{k}}{\partial v^{k}}\left(\frac{v e^{(x+y) v}}{\mu e^{v}-1}\right)+v \frac{e^{y u}}{\lambda e^{u}-1} \frac{\partial^{k}}{\partial v^{k}}\left(\frac{v e^{(x+y) v}}{\mu e^{v}-1}\right)+k \frac{e^{y u}}{\lambda e^{u}-1} \frac{\partial^{k-1}}{\partial v^{k-1}}\left(\frac{v e^{(x+y) v}}{\mu e^{v}-1}\right) \\
&-\lambda v \frac{e^{(1-x) u}}{\lambda e^{u}-1} \frac{\partial^{k}}{\partial v^{k}}\left(\frac{(u+v) e^{(x+y)(u+v)}}{\lambda \mu e^{u+v}-1}\right)-\lambda k \frac{e^{(1-x) u}}{\lambda e^{u}-1} \frac{\partial^{k-1}}{\partial v^{k-1}}\left(\frac{(u+v) e^{(x+y)(u+v)}}{\lambda \mu e^{u+v}-1}\right) .
\end{aligned}
$$

Since $\mathcal{B}_{0}(x ; \lambda)=1$ when $\lambda=1$ and $\mathcal{B}_{0}(x ; \lambda)=0$ when $\lambda \neq 1$ (see, e.g., [8]), so by setting $\mathcal{B}_{0}(x ; \lambda)=\delta_{1, \lambda}$, we get

$$
\frac{e^{x u}}{\lambda e^{u}-1}-\frac{\delta_{1, \lambda}}{u}=\sum_{m=0}^{\infty} \frac{\mathcal{B}_{m+1}(x ; \lambda)}{m+1} \frac{u^{m}}{m !}
$$

On the other hand, by Taylor's theorem, we have

$$
\frac{(u+v) e^{x(u+v)}}{\lambda e^{u+v}-1}=\sum_{n=0}^{\infty} \frac{\partial^{n}}{\partial u^{n}}\left(\frac{u e^{x u}}{\lambda e^{u}-1}\right) \frac{v^{n}}{n !}=\sum_{m=0}^{\infty} \sum_{n=0}^{\infty} \mathcal{B}_{m+n}(x ; \lambda) \frac{u^{m}}{m !} \frac{v^{n}}{n !} .
$$

Applying (2.1), (3.5) and (3.6) to (3.4), in view of the Cauchy product and the complementary addition theorem of the Apostol-Bernoulli polynomials, we derive

$$
\begin{aligned}
\sum_{m=0}^{\infty} & \sum_{n=0}^{\infty}\left[\sum_{i=0}^{n}\left(\begin{array}{l}
n \\
i
\end{array}\right) \sum_{j=0}^{k}\left(\begin{array}{l}
k \\
j
\end{array}\right) \mathcal{B}_{i+j}(x ; \mu) \mathcal{B}_{m+n+k-i-j}(y ; \lambda \mu)\right] \frac{u^{m}}{m !} \frac{v^{n}}{n !} \\
= & \sum_{m=0}^{\infty} \sum_{n=0}^{\infty}\left[\mathcal{B}_{m}(y ; \lambda) \mathcal{B}_{n+k}(x+y ; \mu)+\frac{k \mathcal{B}_{m+1}(y ; \lambda) \mathcal{B}_{n+k-1}(x+y ; \mu)}{m+1}\right] \frac{u^{m}}{m !} \frac{v^{n}}{n !} \\
& +\sum_{m=0}^{\infty} \sum_{n=0}^{\infty} \frac{\mathcal{B}_{m+1}(y ; \lambda) \mathcal{B}_{n+k}(x+y ; \mu)}{m+1} \frac{u^{m}}{m !} \frac{v^{n+1}}{n !}+\frac{k \delta_{1, \lambda}}{u} \sum_{n=0}^{\infty} \mathcal{B}_{n+k-1}(x+y ; \mu) \frac{v^{n}}{n !} \\
& +\frac{\delta_{1, \lambda}}{u} \sum_{n=0}^{\infty} \mathcal{B}_{n+k}(x+y ; \mu) \frac{v^{n+1}}{n !}-\delta_{1, \lambda} \sum_{m=0}^{\infty} \sum_{n=0}^{\infty} \mathcal{B}_{m+n+k}(x+y ; \lambda \mu) \frac{u^{m-1}}{m !} \frac{v^{n+1}}{n !} \\
& +\sum_{m=0}^{\infty} \sum_{n=0}^{\infty}\left[\sum_{i=0}^{m}\left(\begin{array}{c}
m \\
i
\end{array}\right)(-1)^{m+i} \mathcal{B}_{n+k+i}(x+y ; \lambda \mu) \frac{\mathcal{B}_{m+1-i}(x ; \lambda-1)}{m+1-i}\right] \frac{u^{m}}{m !} \frac{v^{n+1}}{n !}
\end{aligned}
$$




$$
\begin{aligned}
& +k \sum_{m=0}^{\infty} \sum_{n=0}^{\infty}\left[\sum_{i=0}^{m}\left(\begin{array}{c}
m \\
i
\end{array}\right)(-1)^{m+i} \mathcal{B}_{n+k+i-1}(x+y ; \lambda \mu) \frac{\mathcal{B}_{m+1-i}\left(x ; \lambda^{-1}\right)}{m+1-i}\right] \frac{u^{m}}{m !} \frac{v^{n}}{n !} \\
& -k \delta_{1, \lambda} \sum_{m=0}^{\infty} \sum_{n=0}^{\infty} \mathcal{B}_{m+n+k-1}(x+y ; \lambda \mu) \frac{u^{m-1}}{m !} \frac{v^{n}}{n !}
\end{aligned}
$$

Comparing the coefficients of $u^{m} v^{n+1} / m !(n+1)$ ! and $u^{m} / m$ ! in (3.7), we conclude our proof.

As a special case of Theorem 3.1, we have the following

Corollary 3.2 Let $m$ and $n$ be positive integers. Then

$$
\begin{gathered}
\frac{1}{m} \sum_{i=0}^{m}\left(\begin{array}{c}
m \\
i
\end{array}\right)(-1)^{m-i} \mathcal{B}_{m-i}\left(y-x ; \lambda^{-1}\right) \mathcal{B}_{n+i-1}(y ; \lambda \mu)-\frac{1}{m} \mathcal{B}_{m}(x ; \lambda) \mathcal{B}_{n-1}(y ; \mu) \\
\quad=-\frac{1}{n} \sum_{i=0}^{n}\left(\begin{array}{c}
n \\
i
\end{array}\right) \mathcal{B}_{n-i}(y-x ; \mu) \mathcal{B}_{m+i-1}(x ; \lambda \mu)+\frac{1}{n} \mathcal{B}_{m-1}(x ; \lambda) \mathcal{B}_{n}(y ; \mu) .
\end{gathered}
$$

Proof Setting $k=0$ and substituting $y$ for $x$ and $x$ for $y$ in Theorem 3.1, we obtain that for non-negative integers $m$ and $n$, we have

$$
\begin{aligned}
\sum_{i=0}^{n}\left(\begin{array}{c}
n \\
i
\end{array}\right) \mathcal{B}_{n-i}(y ; \mu) \mathcal{B}_{m+i}(x ; \lambda \mu) \\
=\mathcal{B}_{m}(x ; \lambda) \mathcal{B}_{n}(x+y ; \mu)+\frac{n}{m+1} \mathcal{B}_{m+1}(x ; \lambda) \mathcal{B}_{n-1}(x+y ; \mu) \\
\quad+\frac{n}{m+1} \sum_{i=0}^{m+1}\left(\begin{array}{c}
m+1 \\
i
\end{array}\right)(-1)^{m+i} \mathcal{B}_{m+1-i}\left(y ; \lambda^{-1}\right) \mathcal{B}_{n+i-1}(x+y ; \lambda \mu) .
\end{aligned}
$$

Hence, replacing $y$ by $y-x$ in the above gives the desired result.

Remark 3.3 Setting $\lambda=\mu=1, x=t$ and $y=1-t$ in Corollary 3.2, by $B_{n}(1-x)=(-1)^{n} B_{n}(x)$ for a non-negative integer $n$, we immediately get the generalization of Woodcock's identity on the classical Bernoulli numbers, see $[18,19]$,

$$
\begin{aligned}
& \frac{1}{m} \sum_{i=0}^{m}\left(\begin{array}{c}
m \\
i
\end{array}\right)(-1)^{i} B_{m-i}(2 t) B_{n+i-1}(t)-\frac{1}{m} B_{m}(t) B_{n-1}(t) \\
& \quad=\frac{1}{n} \sum_{i=0}^{n}\left(\begin{array}{c}
n \\
i
\end{array}\right)(-1)^{i} B_{n-i}(2 t) B_{m+i-1}(t)-\frac{1}{n} B_{n}(t) B_{m-1}(t) \quad(m, n \geq 1) .
\end{aligned}
$$

Applying the difference equation and symmetric distribution of the classical Bernoulli polynomials, one can get $(-1)^{n} B_{n}(-x)=B_{n}(x)+n x^{n-1}$ for a positive integer $n$. Combining with Corollary 3.2, we have another symmetric expression on the classical Bernoulli numbers due to Agoh, see [4, 20],

$$
\frac{1}{m} \sum_{i=1}^{m}\left(\begin{array}{c}
m \\
i
\end{array}\right) B_{m-i} B_{n+i-1}+\frac{1}{n} \sum_{i=1}^{n}\left(\begin{array}{l}
n \\
i
\end{array}\right) B_{n-i} B_{m+i-1}=-B_{m+n-2} \quad(m, n \geq 1)
$$


Using Theorem 3.1, we shall give the following quadratic recurrence formula for Apostol-Bernoulli polynomials.

Theorem 3.4 Let $k, m, n$ be non-negative integers. Then

$$
\begin{aligned}
&\left(\mathcal{B}_{k}(x ; \mu)+\mathcal{B}_{m}(y ; \lambda \mu)\right)^{n} \\
&=- \delta_{1, \lambda} \frac{k ! m !(n+\delta(k, m)(m+k+1))}{(m+k+1) !} \mathcal{B}_{m+n+k}(x+y ; \lambda \mu) \\
&+\sum_{i=0}^{m+k}(-1)^{m+i}\left\{n\left(\begin{array}{c}
m \\
i
\end{array}\right)-k\left(\begin{array}{c}
m \\
i-1
\end{array}\right)\right\} \mathcal{B}_{n+i-1}(x+y ; \lambda \mu) \frac{\mathcal{B}_{m+k+1-i}\left(x ; \lambda^{-1}\right)}{m+k+1-i} \\
&+\sum_{i=0}^{m+k}(-1)^{k+i}\left\{n\left(\begin{array}{c}
k \\
i
\end{array}\right)-m\left(\begin{array}{c}
k \\
i-1
\end{array}\right)\right\} \mathcal{B}_{n+i-1}(x+y ; \mu) \frac{\mathcal{B}_{m+k+1-i}(y ; \lambda)}{m+k+1-i},
\end{aligned}
$$

where $\delta(k, m)=-1$ when $k=m=0, \delta(k, m)=0$ when $k=0, m \geq 1$ or $m=0, k \geq 1$, and $\delta(k, m)=1$ otherwise.

Proof Setting $k=0$ and substituting $m+k$ for $m$ in Theorem 3.1, we have

$$
\begin{aligned}
\left(\mathcal{B}_{0}(x ; \mu)+\mathcal{B}_{m+k}(y ; \lambda \mu)\right)^{n} & \\
= & -\delta_{1, \lambda} \frac{n \mathcal{B}_{m+n+k}(x+y ; \lambda \mu)}{m+k+1}+\mathcal{B}_{m+k}(y ; \lambda) \mathcal{B}_{n}(x+y ; \mu) \\
& +\frac{n \mathcal{B}_{m+k+1}(y ; \lambda) \mathcal{B}_{n-1}(x+y ; \mu)}{m+k+1}+n \sum_{i=0}^{m+k}\left(\begin{array}{c}
m+k \\
i
\end{array}\right)(-1)^{m+k+i} \\
\quad & \mathcal{B}_{n+i-1}(x+y ; \lambda \mu) \frac{\mathcal{B}_{m+k+1-i}\left(x ; \lambda^{-1}\right)}{m+k+1-i}
\end{aligned}
$$

which is just the case $\delta(k, m)=-1$ or 0 in Theorem 3.4. Next, consider the case $\delta(k, m)=1$. We shall use induction on $k$ in Theorem 3.1 to prove Theorem 3.4. Clearly, the case $k=1$ in Theorem 3.1 gives

$$
\begin{aligned}
&\left(\mathcal{B}_{1}(x ; \mu)+\mathcal{B}_{m}(y ; \lambda \mu)\right)^{n}+\left(\mathcal{B}_{0}(x ; \mu)+\mathcal{B}_{m+1}(y ; \lambda \mu)\right)^{n} \\
&=- \delta_{1, \lambda} \frac{n+1}{m+1} \mathcal{B}_{m+n+1}(x+y ; \lambda \mu) \\
& \quad+\mathcal{B}_{m}(y ; \lambda) \mathcal{B}_{n+1}(x+y ; \mu)+\frac{n+1}{m+1} \mathcal{B}_{m+1}(y ; \lambda) \mathcal{B}_{n}(x+y ; \mu) \\
& \quad+(n+1) \sum_{i=0}^{m}\left(\begin{array}{c}
m \\
i
\end{array}\right)(-1)^{m+i} \mathcal{B}_{n+i}(x+y ; \lambda \mu) \frac{\mathcal{B}_{m+1-i}\left(x ; \lambda^{-1}\right)}{m+1-i} .
\end{aligned}
$$

Noting that for any non-negative integer $k$,

$$
\begin{aligned}
& \sum_{i=0}^{m}\left(\begin{array}{c}
m \\
i
\end{array}\right)(-1)^{m+i} \mathcal{B}_{n+k+i-1}(x+y ; \lambda \mu) \frac{\mathcal{B}_{m+1-i}\left(x ; \lambda^{-1}\right)}{m+1-i} \\
& =\sum_{i=0}^{m+k}\left(\begin{array}{c}
m \\
i-k
\end{array}\right)(-1)^{m+k+i} \mathcal{B}_{n+i-1}(x+y ; \lambda \mu) \frac{\mathcal{B}_{m+k+1-i}\left(x ; \lambda^{-1}\right)}{m+k+1-i} .
\end{aligned}
$$


Hence, substituting (3.13) and (3.15) to (3.14), we get the case $k=1$ of Theorem 3.4. Now assume that Theorem 3.4 holds for all positive integers being less than $k$. From (3.1) and (3.15), we obtain

$$
\begin{aligned}
\left(\mathcal{B}_{k}(x ; \mu)+\mathcal{B}_{m}(y ; \lambda \mu)\right)^{n} & \\
= & -\delta_{1, \lambda} \frac{(n+k) \mathcal{B}_{m+n+k}(x+y ; \lambda \mu)}{m+1} \\
& +\mathcal{B}_{m}(y ; \lambda) \mathcal{B}_{n+k}(x+y ; \mu)+\frac{(n+k) \mathcal{B}_{m+1}(y ; \lambda) \mathcal{B}_{n+k-1}(x+y ; \mu)}{m+1} \\
& +(n+k) \sum_{i=0}^{m+k}\left(\begin{array}{c}
m \\
i-k
\end{array}\right)(-1)^{m+k+i} \mathcal{B}_{n+i-1}(x+y ; \lambda \mu) \frac{\mathcal{B}_{m+k+1-i}\left(x ; \lambda^{-1}\right)}{m+k+1-i} \\
& -\left(\mathcal{B}_{0}(x ; \mu)+\mathcal{B}_{m+k}(y ; \lambda \mu)\right)^{n}-\sum_{j=1}^{k-1}\left(\begin{array}{l}
k \\
j
\end{array}\right)\left(\mathcal{B}_{j}(x ; \mu)+\mathcal{B}_{m+k-j}(y ; \lambda \mu)\right)^{n} .
\end{aligned}
$$

Since (3.12) holds for all positive integers being less than $k$, we have

$$
\begin{aligned}
\sum_{j=1}^{k-1}\left(\begin{array}{l}
k \\
j
\end{array}\right)\left(\mathcal{B}_{j}(x ; \mu)+\mathcal{B}_{m+k-j}(y ; \lambda \mu)\right)^{n} \\
=-\delta_{1, \lambda} \frac{(m+n+k+1) \mathcal{B}_{m+n+k}(x+y ; \lambda \mu)}{m+k+1} \sum_{j=1}^{k-1} \frac{\left(\begin{array}{c}
k \\
j
\end{array}\right)}{\left(\begin{array}{c}
m+k \\
j
\end{array}\right)}+\sum_{i=0}^{m+k}(-1)^{m+i} \sum_{j=1}^{k-1}\left(\begin{array}{l}
k \\
j
\end{array}\right) \\
\quad \times(-1)^{k-j}\left\{n\left(\begin{array}{c}
m+k-j \\
i
\end{array}\right)-j\left(\begin{array}{c}
m+k-j \\
i-1
\end{array}\right)\right\} \mathcal{B}_{n+i-1}(x+y ; \lambda \mu) \frac{\mathcal{B}_{m+k+1-i}\left(x ; \lambda^{-1}\right)}{m+k+1-i} \\
\left.\quad+\sum_{i=0}^{m+k}(-1)^{k+i} \sum_{j=1}^{k-1}\left(\begin{array}{c}
k \\
j
\end{array}\right)(-1)^{k-j}\left\{\begin{array}{l}
j \\
n \\
i
\end{array}\right)-(m+k-j)\left(\begin{array}{c}
j \\
i-1
\end{array}\right)\right\} \\
\quad \times \mathcal{B}_{n+i-1}(x+y ; \mu) \frac{\mathcal{B}_{m+k+1-i}(y ; \lambda)}{m+k+1-i} .
\end{aligned}
$$

For any non-negative integers $i, k, m$,

$$
\begin{aligned}
& \sum_{j=0}^{k} \frac{\left(\begin{array}{c}
k \\
j
\end{array}\right)}{\left(\begin{array}{c}
m+k \\
j
\end{array}\right)}=\frac{m+k+1}{m+1}, \\
& \sum_{j=0}^{k}(-1)^{j}\left(\begin{array}{l}
k \\
j
\end{array}\right)\left(\begin{array}{c}
m+j \\
i
\end{array}\right)=(-1)^{k}\left(\begin{array}{c}
m \\
i-k
\end{array}\right) .
\end{aligned}
$$

It follows from (3.18) that

$$
\begin{aligned}
& \sum_{j=1}^{k-1} \frac{\left(\begin{array}{c}
k \\
j
\end{array}\right)}{\left(\begin{array}{c}
m+k \\
j
\end{array}\right)}=\frac{k}{m+1}-\frac{k ! m !}{(m+k) !}, \\
& \sum_{j=1}^{k-1}(-1)^{k-j}\left(\begin{array}{c}
k \\
j
\end{array}\right)\left(\begin{array}{c}
m+k-j \\
i
\end{array}\right)=(-1)^{k}\left\{\left(\begin{array}{c}
m \\
i-k
\end{array}\right)-\left(\begin{array}{c}
m+k \\
i
\end{array}\right)\right\}-\left(\begin{array}{c}
m \\
i
\end{array}\right),
\end{aligned}
$$




$$
\begin{aligned}
& \sum_{j=1}^{k-1}(-1)^{k-j}\left(\begin{array}{l}
k \\
j
\end{array}\right)\left(\begin{array}{c}
m+k-j \\
i-1
\end{array}\right)=(-1)^{k-1} k\left(\begin{array}{c}
m \\
i-k
\end{array}\right)-k\left(\begin{array}{c}
m \\
i-1
\end{array}\right), \\
& \sum_{j=1}^{k-1}(-1)^{k-j}\left(\begin{array}{l}
k \\
j
\end{array}\right)\left(\begin{array}{l}
j \\
i
\end{array}\right)=\left(\begin{array}{c}
0 \\
i-k
\end{array}\right)-(-1)^{k}\left(\begin{array}{l}
0 \\
i
\end{array}\right)-\left(\begin{array}{l}
k \\
i
\end{array}\right), \\
& \sum_{j=1}^{k-1}(-1)^{k-j}(m+k-j)\left(\begin{array}{l}
k \\
j
\end{array}\right)\left(\begin{array}{c}
j \\
i-1
\end{array}\right) \\
& \quad=-m\left(\begin{array}{c}
k \\
i-1
\end{array}\right)-(-1)^{k}(m+k)\left(\begin{array}{c}
0 \\
i-1
\end{array}\right)+m\left(\begin{array}{c}
0 \\
i-k-1
\end{array}\right)-k\left(\begin{array}{c}
0 \\
i-k
\end{array}\right) .
\end{aligned}
$$

By substituting the above identities (3.19)-(3.23) to (3.17), we get

$$
\begin{aligned}
\sum_{j=1}^{k-1}\left(\begin{array}{c}
k \\
j
\end{array}\right)\left(\mathcal{B}_{j}(x ; \mu)+\mathcal{B}_{m+k-j}(y ; \lambda \mu)\right)^{n} \\
=-\delta_{1, \lambda} \frac{(m+n+k+1) \mathcal{B}_{m+n+k}(x+y ; \lambda \mu)}{m+k+1}\left(\frac{k}{m+1}-\frac{k ! m !}{(m+k) !}\right) \\
\quad-\sum_{i=0}^{m+k}(-1)^{m+i}\left\{n\left(\begin{array}{c}
m \\
i
\end{array}\right)-k\left(\begin{array}{c}
m \\
i-1
\end{array}\right)+(-1)^{k} n\left(\begin{array}{c}
m+k \\
i
\end{array}\right)-(-1)^{k}(n+k)\left(\begin{array}{c}
m \\
i-k
\end{array}\right)\right\} \\
\quad \times \mathcal{B}_{n+i-1}(x+y ; \lambda \mu) \frac{\left.\mathcal{B}_{m+k+1-i}(x ; \lambda)^{-1}\right)}{m+k+1-i} \\
\quad-\sum_{i=0}^{m+k}(-1)^{k+i}\left\{n\left(\begin{array}{c}
k \\
i
\end{array}\right)-m\left(\begin{array}{c}
k \\
i-1
\end{array}\right)\right\} \mathcal{B}_{n+i-1}(x+y ; \mu) \frac{\mathcal{B}_{m+k+1-i}(y ; \lambda)}{m+k+1-i} \\
\quad+\frac{n+k}{m+1} \mathcal{B}_{m+1}(y ; \lambda) \mathcal{B}_{n+k-1}(x+y ; \mu)-\frac{n \mathcal{B}_{m+k+1}(y ; \lambda)}{m+k+1} \mathcal{B}_{n-1}(x+y ; \mu) \\
\quad-\mathcal{B}_{m+k}(y ; \lambda) \mathcal{B}_{n}(x+y ; \mu)+\mathcal{B}_{m}(y ; \lambda) \mathcal{B}_{n+k}(x+y ; \mu) .
\end{aligned}
$$

Thus, putting (3.13) and (3.24) to (3.16) concludes the induction step. This completes the proof of Theorem 3.4 .

Obviously, the cases $\lambda=\mu=1$ and $x=y=0$ in Theorem 3.4 lead to the Agoh-Dilcher quadratic recurrence formula (1.3). We now use Theorem 3.4 to give the following formula on products of the Apostol-Bernoulli polynomials.

Corollary 3.5 Let $m$ and $n$ be positive integers. Then

$$
\begin{aligned}
\mathcal{B}_{m}(x ; \lambda) \mathcal{B}_{n}(y ; \mu)= & n \sum_{i=0}^{m}\left(\begin{array}{c}
m \\
i
\end{array}\right)(-1)^{m-i} \mathcal{B}_{m-i}\left(y-x ; \lambda^{-1}\right) \frac{\mathcal{B}_{n+i}(y ; \lambda \mu)}{n+i} \\
& +m \sum_{i=0}^{n}\left(\begin{array}{c}
n \\
i
\end{array}\right) \mathcal{B}_{n-i}(y-x ; \mu) \frac{\mathcal{B}_{m+i}(x ; \lambda \mu)}{m+i} \\
& -(-1)^{m} \delta_{1, \lambda \mu} \frac{m ! n !}{(m+n) !} \mathcal{B}_{m+n}(y-x ; \mu) .
\end{aligned}
$$


Proof Taking $n=0$ and then substituting $m$ for $k, n$ for $m, \lambda$ for $\mu$ and $\mu$ for $\lambda$ in Theorem 3.4, we obtain that for positive integers $m$ and $n$,

$$
\begin{aligned}
\mathcal{B}_{m}(x ; \lambda) \mathcal{B}_{n}(y ; \lambda \mu)= & n \sum_{i=0}^{m}\left(\begin{array}{c}
m \\
i
\end{array}\right)(-1)^{i} \mathcal{B}_{m-i}(x+y ; \lambda) \frac{\mathcal{B}_{n+i}(y ; \mu)}{n+i} \\
& +m \sum_{i=0}^{n}\left(\begin{array}{c}
n \\
i
\end{array}\right)(-1)^{i} \mathcal{B}_{n-i}(x+y ; \lambda \mu) \frac{\mathcal{B}_{m+i}\left(x ; \mu^{-1}\right)}{m+i} \\
& -\frac{\delta_{1, \mu} m ! n !}{(m+n) !} \mathcal{B}_{m+n}(x+y ; \lambda \mu) .
\end{aligned}
$$

Substituting $1-y$ for $y$ and $\mu^{-1}$ for $\lambda \mu$ in (3.26) and using the complementary addition theorem for Apostol-Bernoulli polynomials, we get our result.

Remark 3.6 The cases $\lambda=\mu=1$ and $x=y$ in Corollary 3.5 together with the fact $B_{1}=-1 / 2$ and $B_{2 n+1}=0$ for a positive integer $n$ (see, e.g., [21]) will yield that for positive integers $m$ and $n$, see $[5,22]$,

$$
B_{m}(x) B_{n}(x)=\sum_{i=0}^{\left[\frac{m+n}{2}\right]}\left\{n\left(\begin{array}{c}
m \\
2 i
\end{array}\right)+m\left(\begin{array}{c}
n \\
2 i
\end{array}\right)\right\} B_{2 i} \frac{B_{m+n-2 i}(x)}{m+n-2 i}+(-1)^{m+1} \frac{m ! n !}{(m+n) !} B_{m+n},
$$

where $[x]$ is the maximum integer less than or equal to the real number $x$.

\section{Recurrence formulae for mixed Apostol-Bernoulli and Apostol-Euler polynomials}

In this section, we shall use the above methods to give some quadratic recurrence formulae for mixed Apostol-Bernoulli and Apostol-Euler polynomials. As in the proof of Theorem 3.4, we need the following summation formula concerning the quadratic recurrence of mixed Apostol-Bernoulli and Apostol-Euler polynomials.

Theorem 4.1 Let $k, m, n$ be non-negative integers. Then

$$
\begin{aligned}
\sum_{i=0}^{n}\left(\begin{array}{c}
n \\
i
\end{array}\right)\left(\mathcal{E}_{i}(x ; \mu)+\mathcal{B}_{m+n-i}(y ; \lambda \mu)\right)^{k} \\
=\sum_{i=0}^{m}\left(\begin{array}{c}
m \\
i
\end{array}\right)(-1)^{m+i} \mathcal{E}_{m-i}\left(x ; \lambda^{-1}\right) \mathcal{B}_{n+k+i}(x+y ; \lambda \mu) \\
\quad-\frac{1}{2}\left\{m \mathcal{E}_{m-1}(y ; \lambda) \mathcal{E}_{n+k}(x+y ; \mu)+(n+k) \mathcal{E}_{m}(y ; \lambda) \mathcal{E}_{n+k-1}(x+y ; \mu)\right\} .
\end{aligned}
$$

Proof Multiplying both sides of the identity

$$
\frac{1}{\lambda e^{u}+1} \frac{1}{\mu e^{v}+1}=\left(\frac{\lambda e^{u}}{\lambda e^{u}+1}-\frac{1}{\mu e^{v}+1}\right) \frac{1}{\lambda \mu e^{u+v}-1}
$$

by $2(u+v) e^{x v+y(u+v)}$, we have

$$
\frac{2 e^{x v}}{\mu e^{v}+1} \frac{(u+v) e^{y(u+v)}}{\lambda \mu e^{u+v}-1}=\lambda \frac{2 e^{(1-x) u}}{\lambda e^{u}+1} \frac{(u+v) e^{(x+y)(u+v)}}{\lambda \mu e^{u+v}-1}-\frac{u+v}{2} \frac{2 e^{y u}}{\lambda e^{u}+1} \frac{2 e^{(x+y) v}}{\mu e^{v}+1} .
$$


Making $k$-times derivative for the above identity (4.3) with respect to $v$, with the help of the Leibniz rule, we get

$$
\begin{aligned}
& \sum_{j=0}^{k}\left(\begin{array}{c}
k \\
j
\end{array}\right) \frac{\partial^{j}}{\partial v^{j}}\left(\frac{2 e^{x v}}{\mu e^{v}+1}\right) \frac{\partial^{k-j}}{\partial v^{k-j}}\left(\frac{(u+v) e^{y(u+v)}}{\lambda \mu e^{u+v}-1}\right) \\
&=\lambda \frac{2 e^{(1-x) u}}{\lambda e^{u}+1} \frac{\partial^{k}}{\partial v^{k}}\left(\frac{(u+v) e^{(x+y)(u+v)}}{\lambda \mu e^{u+v}-1}\right) \\
&-\frac{u+v}{2} \frac{2 e^{y u}}{\lambda e^{u}+1} \frac{\partial^{k}}{\partial v^{k}}\left(\frac{2 e^{(x+y) v}}{\mu e^{v}+1}\right)-\frac{k}{2} \frac{2 e^{y u}}{\lambda e^{u}+1} \frac{\partial^{k-1}}{\partial v^{k-1}}\left(\frac{2 e^{(x+y) v}}{\mu e^{v}+1}\right) .
\end{aligned}
$$

Applying (2.2) and (3.6) to (4.4), in view of the Cauchy product and the complementary addition theorem for the Apostol-Euler polynomials, we obtain

$$
\begin{aligned}
\sum_{m=0}^{\infty} & \sum_{n=0}^{\infty}\left[\sum_{i=0}^{n}\left(\begin{array}{c}
n \\
i
\end{array}\right) \sum_{j=0}^{k}\left(\begin{array}{l}
k \\
j
\end{array}\right) \mathcal{E}_{i+j}(x ; \mu) \mathcal{B}_{m+n+k-i-j}(y ; \lambda \mu)\right] \frac{u^{m}}{m !} \cdot \frac{v^{n}}{n !} \\
= & \sum_{m=0}^{\infty} \sum_{n=0}^{\infty}\left[\sum_{i=0}^{m}\left(\begin{array}{c}
m \\
i
\end{array}\right)(-1)^{m+i} \mathcal{E}_{m-i}\left(x ; \lambda^{-1}\right) \mathcal{B}_{n+k+i}(x+y ; \lambda \mu)\right] \frac{u^{m}}{m !} \frac{v^{n}}{n !} \\
& -\sum_{m=0}^{\infty} \sum_{n=0}^{\infty} \frac{\mathcal{E}_{m}(y ; \lambda) \mathcal{E}_{n+k}(x+y ; \mu)}{2} \frac{u^{m+1}}{m !} \frac{v^{n}}{n !}-\sum_{m=0}^{\infty} \sum_{n=0}^{\infty} \frac{\mathcal{E}_{m}(y ; \lambda) \mathcal{E}_{n+k}(x+y ; \mu)}{2} \frac{u^{m}}{m !} \frac{v^{n+1}}{n !} \\
& -\frac{k}{2} \sum_{m=0}^{\infty} \sum_{n=0}^{\infty} \mathcal{E}_{m}(y ; \lambda) \mathcal{E}_{n+k-1}(x+y ; \mu) \frac{u^{m}}{m !} \frac{v^{n}}{n !} .
\end{aligned}
$$

Thus, by comparing the coefficients of $u^{m+1} v^{n+1} /(m+1) !(n+1)$ ! and $u^{m+1} /(m+1)$ ! in (4.5), we complete the proof of Theorem 4.1 .

We now give a special case of Theorem 4.1. We have the following

Corollary 4.2 Let $m$ and $n$ be positive integers. Then

$$
\begin{gathered}
\sum_{i=0}^{m}\left(\begin{array}{c}
m \\
i
\end{array}\right)(-1)^{m-i} \mathcal{E}_{m-i}\left(y-x ; \lambda^{-1}\right) \mathcal{B}_{n+i}(y ; \lambda \mu)-\frac{m}{2} \mathcal{E}_{m-1}(x ; \lambda) \mathcal{E}_{n}(y ; \mu) \\
=\sum_{i=0}^{n}\left(\begin{array}{c}
n \\
i
\end{array}\right) \mathcal{E}_{n-i}(y-x ; \mu) \mathcal{B}_{m+i}(x ; \lambda \mu)-\frac{n}{2} \mathcal{E}_{n-1}(y ; \mu) \mathcal{E}_{m}(x ; \lambda) .
\end{gathered}
$$

Proof Setting $k=0$ and substituting $y$ for $x$ and $x$ for $y$ in Theorem 4.1, we obtain that for positive integers $m$ and $n$,

$$
\begin{aligned}
\sum_{i=0}^{n}\left(\begin{array}{c}
n \\
i
\end{array}\right) \mathcal{E}_{n-i}(y ; \mu) \mathcal{B}_{m+i}(x ; \lambda \mu) \\
=\sum_{i=0}^{m}\left(\begin{array}{c}
m \\
i
\end{array}\right)(-1)^{m-i} \mathcal{E}_{m-i}\left(y ; \lambda^{-1}\right) \mathcal{B}_{n+i}(x+y ; \lambda \mu) \\
\quad-\frac{1}{2}\left\{m \mathcal{E}_{m-1}(x ; \lambda) \mathcal{E}_{n}(x+y ; \mu)+n \mathcal{E}_{n-1}(x+y ; \mu) \mathcal{E}_{m}(x ; \lambda)\right\} .
\end{aligned}
$$

Thus, replacing $y$ by $y-x$ in the above gives the desired result. 
In particular, setting $\lambda=\mu=1, x=t$ and $y=1-t$ in Corollary 4.2 , by the fact $E_{n}(1-$ $x)=(-1)^{n} E_{n}(x)$ for a non-negative integer $n$, we obtain the following symmetric identity involving the Bernoulli and Euler polynomials, see [19].

Corollary 4.3 Let $m$ and $n$ be positive integers. Then

$$
\begin{aligned}
& \sum_{i=0}^{m}\left(\begin{array}{c}
m \\
i
\end{array}\right)(-1)^{i} E_{m-i}(2 t) B_{n+i}(t)-\frac{m}{2} E_{m-1}(t) E_{n}(t) \\
& =\sum_{i=0}^{n}\left(\begin{array}{c}
n \\
i
\end{array}\right)(-1)^{i} E_{n-i}(2 t) B_{m+i}(t)-\frac{n}{2} E_{n-1}(t) E_{m}(t) .
\end{aligned}
$$

Now we apply Theorem 4.1 to give the following recurrence formula for mixed ApostolBernoulli and Apostol-Euler polynomials.

Theorem 4.4 Let $k, m, n$ be non-negative integers. Then

$$
\begin{aligned}
\left(\mathcal{E}_{k}(x ; \mu)\right. & \left.+\mathcal{B}_{m}(y ; \lambda \mu)\right)^{n} \\
= & \sum_{i=0}^{m+k}\left(\begin{array}{c}
m \\
i
\end{array}\right)(-1)^{m+i} \mathcal{B}_{n+i}(x+y ; \lambda \mu) \mathcal{E}_{m+k-i}\left(x ; \lambda^{-1}\right) \\
& \quad-\frac{1}{2} \sum_{i=0}^{m+k}(-1)^{k+i}\left\{n\left(\begin{array}{c}
k \\
i
\end{array}\right)-m\left(\begin{array}{c}
k \\
i-1
\end{array}\right)\right\} \mathcal{E}_{n-1+i}(x+y ; \mu) \mathcal{E}_{m+k-i}(y ; \lambda) .
\end{aligned}
$$

Proof The proof is similar to that of Theorem 3.4, and therefore we leave out some of the more obvious details. Clearly, the case $k=0$ in Theorem 4.4 is complete. Next, consider the case $k \geq 1$ in Theorem 4.4. Assume that Theorem 4.4 holds for all positive integers being less than $k$. In light of (4.1), we have

$$
\begin{aligned}
\left(\mathcal{E}_{k}(x ; \mu)+\mathcal{B}_{m}(y ; \lambda \mu)\right)^{n} & \\
=- & \frac{1}{2}\left\{m \mathcal{E}_{m-1}(y ; \lambda) \mathcal{E}_{n+k}(x+y ; \mu)+(n+k) \mathcal{E}_{m}(y ; \lambda) \mathcal{E}_{n+k-1}(x+y ; \mu)\right\} \\
& +\sum_{i=0}^{m+k}\left(\begin{array}{c}
m \\
i-k
\end{array}\right)(-1)^{m+k+i} \mathcal{B}_{n+i}(x+y ; \lambda \mu) \mathcal{E}_{m+k-i}\left(x ; \lambda^{-1}\right) \\
& -\sum_{j=0}^{k-1}\left(\begin{array}{c}
k \\
j
\end{array}\right)\left(\mathcal{E}_{j}(x ; \mu)+\mathcal{B}_{m+k-j}(y ; \lambda \mu)\right)^{n} .
\end{aligned}
$$

It follows from (4.9) and (4.10) that

$$
\begin{aligned}
\left(\mathcal{E}_{k}(x ; \mu)+\mathcal{B}_{m}(y ; \lambda \mu)\right)^{n} & \\
= & -\frac{1}{2}\left\{m \mathcal{E}_{m-1}(y ; \lambda) \mathcal{E}_{n+k}(x+y ; \mu)+(n+k) \mathcal{E}_{m}(y ; \lambda) \mathcal{E}_{n+k-1}(x+y ; \mu)\right\} \\
& +\sum_{i=0}^{m+k}(-1)^{m+i} \mathcal{B}_{n+i}(x+y ; \lambda \mu) \mathcal{E}_{m+k-i}\left(x ; \lambda^{-1}\right) \sum_{j=0}^{k-1}(-1)^{k-1-j}\left(\begin{array}{c}
k \\
j
\end{array}\right)\left(\begin{array}{c}
m+k-j \\
i
\end{array}\right)
\end{aligned}
$$




$$
\begin{aligned}
& -\frac{1}{2} \sum_{i=0}^{m+k}(-1)^{k+i} \mathcal{E}_{n-1+i}(x+y ; \mu) \mathcal{E}_{m+k-i}(y ; \lambda) \\
& \times \sum_{j=0}^{k-1}(-1)^{k-1-j}\left\{n\left(\begin{array}{c}
k \\
j
\end{array}\right)\left(\begin{array}{l}
j \\
i
\end{array}\right)-(m+k-j)\left(\begin{array}{c}
k \\
j
\end{array}\right)\left(\begin{array}{c}
j \\
i-1
\end{array}\right)\right\} \\
& +\sum_{i=0}^{m+k}\left(\begin{array}{c}
m \\
i-k
\end{array}\right)(-1)^{m+k+i} \mathcal{E}_{m+k-i}\left(x ; \lambda^{-1}\right) \mathcal{B}_{n+i}(x+y ; \mu) .
\end{aligned}
$$

In view of (3.19), (3.21) and (3.22), we have

$$
\begin{aligned}
& \sum_{j=0}^{k-1}(-1)^{k-1-j}\left(\begin{array}{l}
k \\
j
\end{array}\right)\left(\begin{array}{c}
m+k-j \\
i
\end{array}\right)=(-1)^{k-1}\left(\begin{array}{c}
m \\
i-k
\end{array}\right)+\left(\begin{array}{c}
m \\
i
\end{array}\right), \\
& \sum_{j=0}^{k-1}(-1)^{k-1-j}\left(\begin{array}{l}
k \\
j
\end{array}\right)\left(\begin{array}{l}
j \\
i
\end{array}\right)=-\left(\begin{array}{c}
0 \\
i-k
\end{array}\right)+\left(\begin{array}{l}
k \\
i
\end{array}\right), \\
& \sum_{j=0}^{k-1}(-1)^{k-1-j}(k+m-j)\left(\begin{array}{c}
k \\
j
\end{array}\right)\left(\begin{array}{c}
j \\
i-1
\end{array}\right)=m\left(\begin{array}{c}
k \\
i-1
\end{array}\right)-m\left(\begin{array}{c}
0 \\
i-k-1
\end{array}\right)+k\left(\begin{array}{c}
0 \\
i-k
\end{array}\right) .
\end{aligned}
$$

Thus, applying (4.12), (4.13) and (4.14) to (4.11), we conclude the induction step. This completes the proof of Theorem 4.4 .

Corollary 4.5 Let $m$ be a non-negative integer and $n$ be a positive integer. Then

$$
\begin{aligned}
\mathcal{E}_{m}(x ; \lambda) \mathcal{B}_{n}(y ; \mu)= & \frac{n}{2} \sum_{i=0}^{m}\left(\begin{array}{c}
m \\
i
\end{array}\right)(-1)^{m-i} \mathcal{E}_{m-i}\left(y-x ; \lambda^{-1}\right) \mathcal{E}_{n+i-1}(y ; \lambda \mu) \\
& +\sum_{i=0}^{n}\left(\begin{array}{c}
n \\
i
\end{array}\right) \mathcal{B}_{n-i}(y-x ; \mu) \mathcal{E}_{m+i}(x ; \lambda \mu) .
\end{aligned}
$$

Proof Taking $n=0$ in Theorem 4.4, and then substituting $m$ for $k, n$ for $m, \lambda$ for $\mu$ and $\mu$ for $\lambda$, we have

$$
\begin{aligned}
\mathcal{E}_{m}(x ; \mu) \mathcal{B}_{n}(y ; \lambda \mu)= & -\frac{n}{2} \sum_{i=0}^{m}\left(\begin{array}{c}
m \\
i
\end{array}\right)(-1)^{i} \mathcal{E}_{m-i}(x+y ; \lambda) \mathcal{E}_{n+i-1}(y ; \mu) \\
& +\sum_{i=0}^{n}\left(\begin{array}{c}
n \\
i
\end{array}\right)(-1)^{i} \mathcal{B}_{n-i}(x+y ; \lambda \mu) \mathcal{E}_{m+i}\left(x ; \mu^{-1}\right) .
\end{aligned}
$$

By substituting $1-y$ for $y$ and $\mu^{-1}$ for $\lambda \mu$ in (4.16), in view of the complementary addition theorems of the Apostol-Bernoulli and Apostol-Euler polynomials, the desired result follows immediately.

In order to give the quadratic recurrence formula for the Apostol-Euler polynomials, it is routine to present a summation formula concerning the quadratic recurrence of the Apostol-Euler polynomials. 
Theorem 4.6 Let $k, m, n$ be non-negative integers. Then

$$
\begin{aligned}
\sum_{i=0}^{n}\left(\begin{array}{c}
n \\
i
\end{array}\right)\left(\mathcal{E}_{i}(x ; \mu)+\mathcal{E}_{m+n-i}(y ; \lambda \mu)\right)^{k} \\
=-2 \sum_{i=0}^{m}\left(\begin{array}{c}
m \\
i
\end{array}\right)(-1)^{m+i} \mathcal{E}_{n+k+i}(x+y ; \lambda \mu) \frac{\mathcal{B}_{m+1-i}\left(x ; \lambda^{-1}\right)}{m+1-i} \\
\quad+\frac{2}{m+1}\left\{\delta_{1, \lambda} \mathcal{E}_{m+n+k+1}(x+y ; \lambda \mu)-\mathcal{B}_{m+1}(y ; \lambda) \mathcal{E}_{n+k}(x+y ; \mu)\right\}
\end{aligned}
$$

Proof Multiplying both sides of the identity

$$
\frac{1}{\lambda e^{u}-1} \frac{1}{\mu e^{v}+1}=\left(\frac{\lambda e^{u}}{\lambda e^{u}-1}-\frac{1}{\mu e^{v}+1}\right) \frac{1}{\lambda \mu e^{u+v}+1}
$$

by $4 e^{x v+y(u+v)}$, we obtain

$$
\frac{2 e^{x v}}{\mu e^{v}+1} \frac{2 e^{y(u+v)}}{\lambda \mu e^{u+v}+1}=2 \lambda \frac{e^{(1-x) u}}{\lambda e^{u}-1} \frac{2 e^{(x+y)(u+v)}}{\lambda \mu e^{u+v}+1}-2 \frac{e^{y u}}{\lambda e^{u}-1} \frac{2 e^{(x+y) v}}{\mu e^{v}+1} .
$$

Making $k$-times derivative for the above identity (4.19) with respect to $v$, with the help of the Leibniz rule, we derive

$$
\begin{aligned}
& \sum_{j=0}^{k}\left(\begin{array}{l}
k \\
j
\end{array}\right) \frac{\partial^{j}}{\partial v^{j}}\left(\frac{2 e^{x v}}{\mu e^{v}+1}\right) \frac{\partial^{k-j}}{\partial v^{k-j}}\left(\frac{2 e^{y(u+v)}}{\lambda \mu e^{u+v}+1}\right) \\
& \quad=2 \lambda \frac{e^{(1-x) u}}{\lambda e^{u}-1} \frac{\partial^{k}}{\partial v^{k}}\left(\frac{2 e^{(x+y)(u+v)}}{\lambda \mu e^{u+v}+1}\right)-2 \frac{e^{y u}}{\lambda e^{u}-1} \frac{\partial^{k}}{\partial v^{k}}\left(\frac{2 e^{(x+y) v}}{\mu e^{v}+1}\right) .
\end{aligned}
$$

Note that from Taylor's theorem, we have

$$
\frac{2 e^{x(u+v)}}{\lambda e^{u+v}+1}=\sum_{n=0}^{\infty} \frac{\partial^{n}}{\partial u^{n}}\left(\frac{2 e^{x u}}{\lambda e^{u}+1}\right) \frac{v^{n}}{n !}=\sum_{m=0}^{\infty} \sum_{n=0}^{\infty} \mathcal{E}_{m+n}(x ; \lambda) \frac{u^{m}}{m !} \cdot \frac{v^{n}}{n !}
$$

Applying (2.2), (3.5) and (4.21) to (4.20), in view of the Cauchy product and the complementary addition theorem for the Apostol-Bernoulli polynomials, we obtain

$$
\begin{aligned}
\sum_{m=0}^{\infty} \sum_{n=0}^{\infty}\left[\sum_{i=0}^{n}\left(\begin{array}{l}
n \\
i
\end{array}\right) \sum_{j=0}^{k}\left(\begin{array}{l}
k \\
j
\end{array}\right) \mathcal{E}_{i+j}(x ; \mu) \mathcal{E}_{m+n+k-i-j}(y ; \lambda \mu)\right] \frac{u^{m}}{m !} \frac{v^{n}}{n !} \\
=-2 \sum_{m=0}^{\infty} \sum_{n=0}^{\infty}\left[\sum_{i=0}^{m}\left(\begin{array}{c}
m \\
i
\end{array}\right)(-1)^{m+i} \mathcal{E}_{n+k+i}(x+y ; \lambda \mu) \frac{\mathcal{B}_{m+1-i}\left(x ; \lambda^{-1}\right)}{m+1-i}\right] \frac{u^{m}}{m !} \frac{v^{n}}{n !} \\
-2 \sum_{m=0}^{\infty} \sum_{n=0}^{\infty} \mathcal{E}_{n+k}(x+y ; \mu) \frac{\mathcal{B}_{m+1}(y ; \lambda)}{m+1} \frac{u^{m}}{m !} \frac{v^{n}}{n !} \\
+2 \delta_{1, \lambda} \sum_{m=0}^{\infty} \sum_{n=0}^{\infty} \mathcal{E}_{m+n+k}(x+y ; \lambda \mu) \frac{u^{m-1}}{m !} \frac{v^{n}}{n !}-\frac{2 \delta_{1, \lambda}}{u} \sum_{n=0}^{\infty} \mathcal{E}_{n+k}(x+y ; \mu) \frac{v^{n}}{n !} .
\end{aligned}
$$

Thus, comparing the coefficients of $u^{m} v^{n} / m ! n !$ in (4.22) gives Theorem 4.6. 
Remark 4.7 Setting $k=0$ in Theorem 4.6, one can easily reobtain Corollary 4.5.

Theorem 4.8 Let $k, m, n$ be non-negative integers. Then

$$
\begin{aligned}
\left(\mathcal{E}_{k}(x ; \mu)+\mathcal{E}_{m}(y ; \lambda \mu)\right)^{n} & \\
= & 2 \delta_{1, \lambda} \frac{k ! m !}{(m+k+1) !} \mathcal{E}_{m+n+k+1}(x+y ; \lambda \mu) \\
& -2 \sum_{i=0}^{m+k}(-1)^{m+i}\left(\begin{array}{c}
m \\
i
\end{array}\right) \mathcal{E}_{n+i}(x+y ; \lambda \mu) \frac{\mathcal{B}_{m+k+1-i}\left(x ; \lambda^{-1}\right)}{m+k+1-i} \\
& -2 \sum_{i=0}^{m+k}(-1)^{k+i}\left(\begin{array}{c}
k \\
i
\end{array}\right) \mathcal{E}_{n+i}(x+y ; \mu) \frac{\mathcal{B}_{m+k+1-i}(y ; \lambda)}{m+k+1-i} .
\end{aligned}
$$

Proof Clearly, the case $k=0$ in Theorem 4.6 leads to the case $k=0$ in Theorem 4.8. Now consider the case $k \geq 1$ in Theorem 4.8. Assume that Theorem 4.8 holds for all positive integers being less than $k$. By (4.17) we have

$$
\begin{aligned}
\left(\mathcal{E}_{k}(x ; \mu)+\mathcal{E}_{m}(y ; \lambda \mu)\right)^{n} & \\
= & \frac{2}{m+1}\left\{\delta_{1, \lambda} \mathcal{E}_{m+n+k+1}(x+y ; \lambda \mu)-\mathcal{B}_{m+1}(y ; \lambda) \mathcal{E}_{n+k}(x+y ; \mu)\right\} \\
& -2 \sum_{i=0}^{m+k}\left(\begin{array}{c}
m \\
i-k
\end{array}\right)(-1)^{m+k+i} \mathcal{E}_{n+i}(x+y ; \lambda \mu) \frac{\mathcal{B}_{m+k+1-i}\left(x ; \lambda^{-1}\right)}{m+k+1-i} \\
& -\sum_{j=0}^{k-1}\left(\begin{array}{c}
k \\
j
\end{array}\right)\left(\mathcal{E}_{j}(x ; \mu)+\mathcal{E}_{m+k-j}(y ; \lambda \mu)\right)^{n} .
\end{aligned}
$$

It follows from (4.23) and (4.24) that

$$
\begin{aligned}
\left(\mathcal{E}_{k}(x ; \mu)+\right. & \left.\mathcal{E}_{m}(y ; \lambda \mu)\right)^{n} \\
= & \frac{2}{m+1}\left\{\mathcal{E}_{m+n+k+1}(x+y ; \lambda \mu)-\mathcal{B}_{m+1}(y ; \lambda) \mathcal{E}_{n+k}(x+y ; \mu)\right\} \\
& -2 \sum_{i=0}^{m+k}\left(\begin{array}{c}
m \\
i-k
\end{array}\right)(-1)^{m+k+i} \mathcal{E}_{n+i}(x+y ; \lambda \mu) \frac{\mathcal{B}_{m+k+1-i}\left(x ; \lambda^{-1}\right)}{m+k+1-i} \\
& -2 \delta_{1, \lambda} \frac{\mathcal{E}_{m+n+k+1}(x+y ; \lambda \mu)}{k+m+1} \sum_{j=0}^{k-1} \frac{\left(\begin{array}{c}
k \\
j
\end{array}\right)}{\left(\begin{array}{c}
m+k \\
j
\end{array}\right)}-2 \sum_{i=0}^{m+k}(-1)^{m+i} \mathcal{E}_{n+i}(x+y ; \lambda \mu) \\
& \times \frac{\mathcal{B}_{m+k+1-i}\left(x ; \lambda^{-1}\right)}{m+k+1-i} \sum_{j=0}^{k-1}(-1)^{k-1-j}\left(\begin{array}{c}
k \\
j
\end{array}\right)\left(\begin{array}{c}
m+k-j \\
i
\end{array}\right) \\
& -2 \sum_{i=0}^{m+k}(-1)^{k+i} \mathcal{E}_{n+i}(x+y ; \mu) \frac{\mathcal{B}_{m+k+1-i}(y ; \lambda)}{m+k+1-i} \sum_{j=0}^{k-1}(-1)^{k-1-j}\left(\begin{array}{l}
k \\
j
\end{array}\right)\left(\begin{array}{l}
j \\
i
\end{array}\right) .
\end{aligned}
$$

Thus, applying (3.18), (4.12) and (4.13) to (4.25), we conclude the induction step. This completes the proof of Theorem 4.8 . 
Corollary 4.9 Let $m$ and $n$ be non-negative integers. Then

$$
\begin{aligned}
\mathcal{E}_{m}(x ; \lambda) \mathcal{E}_{n}(y ; \mu)= & 2 \sum_{i=0}^{m}\left(\begin{array}{c}
m \\
i
\end{array}\right)(-1)^{m-i} \mathcal{E}_{m-i}\left(y-x ; \lambda^{-1}\right) \frac{\mathcal{B}_{n+1+i}(y ; \lambda \mu)}{n+1+i} \\
& -2 \sum_{i=0}^{n}\left(\begin{array}{c}
n \\
i
\end{array}\right) \mathcal{E}_{n-i}(y-x ; \mu) \frac{\mathcal{B}_{m+1+i}(x ; \lambda \mu)}{m+1+i} \\
& -2 \delta_{1, \lambda \mu} \frac{(-1)^{m} m ! n !}{(m+n+1) !} \mathcal{E}_{m+n+1}\left(y-x ; \lambda^{-1}\right)
\end{aligned}
$$

Proof Taking $n=0$ in Theorem 4.8, and substituting $m$ for $k, n$ for $m, \lambda$ for $\mu$ and $\mu$ for $\lambda$, we get

$$
\begin{aligned}
\mathcal{E}_{m}(x ; \lambda) \mathcal{E}_{n}(y ; \lambda \mu)= & 2 \delta_{1, \mu} \frac{m ! n !}{(m+n+1) !} \mathcal{E}_{m+n+1}(x+y ; \lambda \mu) \\
& -2 \sum_{i=0}^{m}\left(\begin{array}{c}
m \\
i
\end{array}\right)(-1)^{i} \mathcal{E}_{m-i}(x+y ; \lambda \mu) \frac{\mathcal{B}_{n+1+i}\left(x ; \mu^{-1}\right)}{n+1+i} \\
& -2 \sum_{i=0}^{n}\left(\begin{array}{c}
n \\
i
\end{array}\right)(-1)^{i} \mathcal{E}_{n-i}(x+y ; \lambda) \frac{\mathcal{B}_{m+1+i}(y ; \mu)}{m+1+i} .
\end{aligned}
$$

Hence, substituting $1-y$ for $y$ and $\mu^{-1}$ for $\lambda \mu$ in (4.27), in view of the complementary addition theorems of the Apostol-Bernoulli and Apostol-Euler polynomials, the desired result follows immediately.

Remark 4.10 The cases $\lambda=\mu=1$ and $x=y$ in Corollaries 4.5 and 4.9 will yield the corresponding formulae for $B_{m}(x) E_{n}(x)$ and $E_{m}(x) E_{n}(x)$ presented in [5]. We leave them to the interested readers for an exercise. For different proofs of Corollaries 3.5, 4.5 and 4.9, we refer to [23].

\section{Competing interests}

The authors declare that they have no competing interests.

\section{Authors' contributions}

All authors contributed equally and significantly in writing this paper.

\section{Acknowledgements}

The authors express their gratitude to the referees for careful reading and helpful comments on the previous version of this work. This work is supported by the National Natural Science Foundation of China (Grant No. 11071194).

\section{Received: 29 August 2012 Accepted: 21 November 2012 Published: 10 December 2012}

\section{References}

1. Cohen, H: Number Theory. Volume II. Analytic and Modern Tools. Graduate Texts in Math., vol. 240. Springer, New York (2007)

2. Nörlund, NE: Vorlesungen über Differnzenrechnung. Springer, Berlin (1924)

3. Srivastava, HM, Choi, J: Series Associated with the Zeta and Related Functions. Kluwer Academic, Dordrecht (2001)

4. Agoh, T, Dilcher, K: Convolution identities and lacunary recurrences for Bernoulli numbers. J. Number Theory 124, 105-122 (2007)

5. Nielsen, N: In: Traité élémentaire des nombres de Bernoulli, pp. 75-78. Gauthier-Villars, Paris (1923)

6. Apostol, TM: On the Lerch zeta function. Pac. J. Math. 1, 161-167 (1951)

7. Srivastava, HM: Some formulas for the Bernoulli and Euler polynomials at rational arguments. Math. Proc. Camb. Philos. Soc. 129, 77-84 (2000)

8. Luo, QM, Srivastava, HM: Some generalizations of the Apostol-Bernoulli and Apostol-Euler polynomials. J. Math. Anal. Appl. 308, 290-302 (2005) 
9. Luo, QM: Apostol-Euler polynomials of higher order and Gaussian hypergeometric functions. Taiwan. J. Math. 10, 917-925 (2006)

10. Boyadzhiev, KN: Apostol-Bernoulli functions, derivative polynomials and Eulerian polynomials. Adv. Appl. Discrete Math. 1, 109-122 (2008)

11. Garg, M, Jain, K, Srivastava, HM: Some relationships between the generalized Apostol-Bernoulli polynomials and Hurwitz-Lerch zeta functions. Integral Transforms Spec. Funct. 17, 803-815 (2006)

12. Kim, MS, Hu, S: Sums of products of Apostol-Bernoulli numbers. Ramanujan J. 28, 113-123 (2012)

13. Luo, QM: Fourier expansions and integral representations for the Apostol-Bernoulli and Apostol-Euler polynomials. Math. Comput. 78, 2193-2208 (2009)

14. Luo, QM, Srivastava, HM: Some relationships between the Apostol-Bernoulli and Apostol-Euler polynomials. Comput. Math. Appl. 51, 631-642 (2006)

15. Luo, QM: The multiplication formulas for the Apostol-Bernoulli and Apostol-Euler polynomials of higher order. Integral Transforms Spec. Funct. 20, 377-391 (2009)

16. Prévost, M: Padé approximation and Apostol-Bernoulli and Apostol-Euler polynomials. J. Comput. Appl. Math. 233, 3005-3017 (2010)

17. Srivastava, HM, Todorov, PG: An explicit formula for the generalized Bernoulli polynomials. J. Math. Anal. Appl. 130, 509-513 (1988)

18. He, Y, Zhang, WP: Some sum relations involving Bernoulli and Euler polynomials. Integral Transforms Spec. Funct. 22, 207-215 (2011)

19. Pan, H, Sun, ZW: New identities involving Bernoulli and Euler polynomials. J. Comb. Theory, Ser. A 11, 156-175 (2006)

20. Agoh, T: On Bernoulli numbers. I. C. R. Math. Acad. Sci. Soc. R. Can. 10, 7-12 (1988)

21. Abramowitz, M, Stegun, IA: Handbook of Mathematical Functions. Dover, New York (1972)

22. Carlitz, L: Note on the integral of the product of several Bernoulli polynomials. J. Lond. Math. Soc. 34, 361-363 (1959)

23. He, Y, Wang, CP: Some formulae of products of the Apostol-Bernoulli and Apostol-Euler polynomials. Discrete Dyn. Nat. Soc. 2012, Article ID 927953 (2012)

doi:10.1186/1687-1847-2012-209

Cite this article as: He and Wang: Recurrence formulae for Apostol-Bernoulli and Apostol-Euler polynomials. Advances in Difference Equations 2012 2012:209.

\section{Submit your manuscript to a SpringerOpen ${ }^{\circ}$ journal and benefit from:}

- Convenient online submission

Rigorous peer review

- Immediate publication on acceptance

- Open access: articles freely available online

- High visibility within the field

- Retaining the copyright to your article 